Review Article

\title{
COVID- I 9 Vaccine Development and Introduction - A Review of Regulatory Process
}

\section{Varun Singh}

B.Pharm, MBA (Healthcare Management), Public Health Expert.

Former Consultant (Urban Health and RCH Division), Ministry of Health and Family Welfare (Government of India), New Delhi. DOI: https://doi.org/10.24321/2455.7048.202023

\section{I $\quad \mathbf{N} \quad \mathbf{F} \quad \mathbf{O}$}

E-mail Id:

varunsingh.mohfw@gmail.com

Orcid Id:

https://orcid.org/0000-0003-0264-1576

How to cite this article:

Singh V. COVID-19 Vaccine Development and Introduction - A Review of Regulatory Process. Epidem Int 2020; 5(3): 19-27.

Date of Submission: 2020-08-05 Date of Acceptance: 2020-08-27

\section{$\begin{array}{llllllll}\mathbf{A} & \mathbf{B} & \mathbf{S} & \mathbf{T} & \mathbf{R} & \mathbf{A} & \mathbf{C} & \mathbf{T}\end{array}$}

COVID-19 is the infectious disease caused by a novel strain of coronavirus (SARS-CoV-2) reported first in Wuhan, China, in December 2019. Coronaviruses are a family of viruses that causes illnesses like a common cold, MERS (Middle East Respiratory Syndrome), SARS (Severe Acute Respiratory Syndrome), etc. After the US and Brazil, India has reached the third rank in the world in terms of the high number of COVID-19 cases. To protect humans from the disease, a race has begun among vaccine developers around the world and hundreds of COVID-19 vaccine candidates are at various phases of the clinical trial. Many of us may not be aware about technical terms like clinical trial, compassionate use, emergency use etc., as well as various regulatory process involved. Therefore, the article is an attempt to chalk-out various concepts that are presently resonating with respect to COVID-19 vaccine development and introduction with few global examples. The article also aims to depicts the procedures for new vaccine approval \& introduction process in India.

Keywords: COVID-19, SARS-CoV-2, New Vaccine Introduction, Emergency Use Authorization (EUA), Compassionate Use, Clinical Trials

\section{Introduction}

The COVID-19 pandemic has adversely affected the overall health status of the population worldwide and may lead to long-term economic slowdown. In India, the Pandemic has resulted in total reported cases of 1.53 million and deaths of .03 million people that are increasing day by day. ${ }^{1}$ Everyone including government departments, policymakers, donor \& partner agencies, manufacturers, scientists, researchers, administrators, regulators and the common public are striving hard to get proven protection measures \& treatment against the SARS-CoV-2 agent but unfortunately, a safe vaccine with proven efficacy, quality \& safety and regulatory approval is not available yet. During the lockdown, we all are listening to new terms in audio-visual \& print media such as clinical trials, compassionate use/ expanded access and emergency use authorization, and many more but may have limited information about these concepts. In the current COVID-19 scenario, many of us may have questions in mind -(a) what is a clinical trial, compassionate use, expanded access, and EUA? (b) What are the standard operating procedures for new vaccine introduction and expected approval time in a normal situation? (c) Is it possible to introduce the COVID-19 vaccine, with unproven efficacy, in shorter time-span under EUA or compassionate use? (d) If yes, is it ethical \& safe?

These are obvious and relevant questions. Therefore, to understand these concepts and its importance, the article will briefly explain: (A) Methodology, (B) Clinical Development Stage/ Clinical Trial Phases on Humans, (C) New Vaccine Introduction Process with Examples from US 
and India, (D) Concept of Compassionate Use (Expanded Access), (E) Concept of Emergency Use Authorization and, (F) Discussion.

\section{Methodology}

Desk review of literature, i.e. research articles, published guidelines, and Press Information Bureau (PIB) releases. The Government of India (Gol) guidelines/ reports and by referring to an international document providing information about New Vaccine Introduction (NVI), Clinical Trials, Emergency Use Authorization (EUA), and Compassionate Use.

\section{Clinical Development Stages/ Clinical Trial Phases on Humans}

The vaccine development cycle includes many stages such as exploratory stage, pre-clinical stage, clinical development, regulatory review \& approval, manufacturing, and quality control. However, the article will primarily focus on the clinical development stage, also termed as clinical trial phases on humans. As suggested by US Food and Drug Administration (FDA) certain regulatory approval steps must be followed by the new vaccine development sponsor(s) such as - an investigational new drug application, pre-licensure vaccine clinical trials, a Biologics License Application (BLA), inspection of the manufacturing facility and presentation of findings. Clinical trial begins after the drug enters into the investigational new drug. There are three phases of clinical trials in humans i.e. phase I, phase II, and phase III. In most of the countries, all humansubject studies require formal regulatory approval, ethical clearance, and ethical consideration as per the Declaration of Helsinki i.e. a statement of ethical principles for medical research involving human subjects. ${ }^{2}$

Phase $\mathrm{I}$ is concerned with safety parameters and carried out in a limited number of healthy adults (say 20-100) to test vaccine properties, tolerability, clinical laboratory, and pharmacological parameters. Phase II is carried out to obtain information on the vaccine's ability to produce its desired effects and thus, involve a large number of subjects in the target population. Phase III is undertaken to fully assess the efficacy and safety of a vaccine. Phase III is a pivotal study for getting vaccine/drug license, manufacturing and to apply for market authorization by country's regulatory agency. It is pertinent to note that the new vaccine introduction is a complex phenomenon and a systematic process and usually takes time. ${ }^{3}$

\section{New Vaccine Introduction Process}

The new vaccine introduction process comes after the development of vaccines with proven efficacy \& safety parameters, license, and market authorization. Moreover, there are country-specific processes to add the licensed vaccine in the recommended schedule. For instance, in the United States, the Advisory Committee on Immunization Practices (ACIP) is responsible to review clinical trial data \& other studies, monitor vaccine safety \& effectiveness data before as well as after the use of vaccines. ACIP may recommend or rejects vaccines for routine use. Recommendations made by ACIP could not be treated officially until reviewed \& approved by the Director of the Center for Disease Control (CDC) \& is duly published. ${ }^{4}$

Similarly, In India, the new vaccine introduction process involves many activities, that for better understanding, herein, categorized as - (a) Review of Key Parameters (b) New Vaccine Recommendation and Approval Process (c) Pre-Introduction/ Preparatory Phase (d) Vaccine Introduction Phase/ Vaccine Launch (e) Post-Introduction and Expansion Phase.

\section{Review of Key Parameters}

Before deciding whether it is a need of the hour and worth introducing a new vaccine or not, certain key parameters ${ }^{5}$ need to be considered by the policymakers, these are:

Public Health Priority: The first question before making vaccine decisions is - Whether the disease is a health priority or not? It includes considering disease status, resource availability, on-going priority health schemes, global and national targets, and government's focus on specific public health issues.

Disease Burden: Number of disease cases, morbidity \& mortality rates, disease distribution \& determinants, pandemic/ epidemic status are another critical indicator. For example: adult JE vaccine in India are introduced in identified districts prone to JE outbreak.

Vaccine Efficacy, Quality, and Safety: Ensuring vaccine efficacy, quality and safety are of utmost importance before licensing and making it available for vaccination.

Feasibility using Other Vaccines: Out of available vaccine candidates \& forms, the feasibility of vaccine usage among other available options, to make epidemiological \& microbiological changes over time, maybe reviewed. For instance, a feasibility \& efficacy study on injectable and oral forms of Rota Virus Vaccine (RVV) may have been carried out before phased introduction and expansion.

Economic and Financial Issues: The cost-benefit analysis of introducing new vaccine, program's financial sustainability, and the cost impact of new vaccine introduction on overall health budget are other critical and important criteria for deciding the new vaccine introduction. Financial sustainability i.e. capacity of the government to incur the cost of new vaccine and other associated expenditures 
under health budget plays a crucial role. Fund availability and potential sources of future funding (i.e. from Government, BMGF, GAVI, World Bank, Corporate Social Responsibility funds etc.) helps in deciding initial funding and financial sustainability aspects.

Vaccine Presentation: Vaccine presentation criteria (such as single dose or multi-dose, monovalent, or in combination, liquid/ lyophilized vaccine) is useful to consider the availability of preferred formulation and presentation of a new vaccine. For example: In India, RVV has been introduced in oral \& injectable forms.

Vaccines Manufacturers and Supplier's Availability: Less number of manufactures or a single manufacturer in the global market often leads to less vaccine production capacity \& supply, as well as, high vaccine price due to the non-competitive market. Besides as per the Gol's General Financial Rules (GFR) 2017, at least three suppliers need to take part in the tendering process for vaccine procurement, with exceptions in few critical situation as deemed suitable by the Government. Thus, it makes the availability of manufacturers and suppliers as an important criterion for consideration.

\section{New Vaccine Recommendation \& Approval Process in India}

After considering the key parameters mentioned above, if the policymakers decide to look for new vaccine-related aspects carefully, the approval process gets initiated. The introduction of a new vaccine is an extensive process that involves technical, financial, and administrative reviews, meetings, and approvals at various levels. In India, Technical groups (SAGE, NTAGI, STSC, Vaccine Expert Committee and Vaccine expert group) and Policy-making bodies (EPC, MSG) are responsible for "New Vaccine Introduction". 6,7,8

Brief details of the process are as follows:

Strategic Advisory Group of Experts: The SAGE is the advisory group to WHO constituted in 1999. SAGE advises WHO on overall global policy and decision making.

National Technical Advisory Group on Immunization: In India, NTAGI was established in August 2001 to strengthen the decision making of the new vaccine introduction. It is the apex advisory body of the MoHFW that makes suitable recommendations related to immunization, which include the introduction and expansion of new vaccines, as well as, of vaccines having less coverage. As part of the NTAGI proceedings, the group of independent and multi-disciplinary health experts, as well as ex-officio members, systematically review data on disease burden, vaccine safety, equity, financial sustainability, and logistical implications of the decision to introduce new vaccines in the program and give recommendations concerning the introduction of new vaccines. The role of NTAGI members is mainly consultative and the ultimate decision remains in the hands of the government.

Standing Technical Sub Committee (STSC): The NTAGI was reconstituted with STSC in June 2013 comprising of independent experts. STSC undertakes detailed deliberations on technical issues and provide recommendations to the NTAGI for further review.

NTAGI Secretariat: NTAGI secretariat facilitates the organization of NTAGI and STSC meetings and other associated activities.

Empowered Program Committee (EPC): EPC of National Health Mission is an executive committee formed under the chairpersonship of the Secretary (Health and Family Welfare) having members from linked ministries, NITI Aayog, Directorate General of Health Services(DGHS), Additional Secretary \& Financial Advisor (AS\&FA) and AS (Health \& FW), 2 public health professionals and Additional Secretary \& Mission Director of India's National Health Mission (AS\&MD-NHM) as a convener. The EPC reviews the recommendation of NTAGI in detail, before presenting and recommending the same to Mission Steering Group (MSG) of NHM for review and final approval.

Mission Steering Group (MSG): The MSG is the highest policy-making and steering institution constituted under the chairpersonship of Hon'ble Union Minister of Health \& Family Welfare (Gol). The group comprises members from linked ministries, DGHS, NITI Aayog, 4 secretaries from high focus states, AS\&FA, AS (Health \& FW), 9 public health professionals, and Secretary (HFW) as a convener. MSG's recommendations are considered as final official approval for introducing new vaccines in the Country. Once the MSG approves NTAGI's recommendation, the constitution of the Vaccine Expert Committee carried out to develop an effective strategy for the new vaccine introduction.

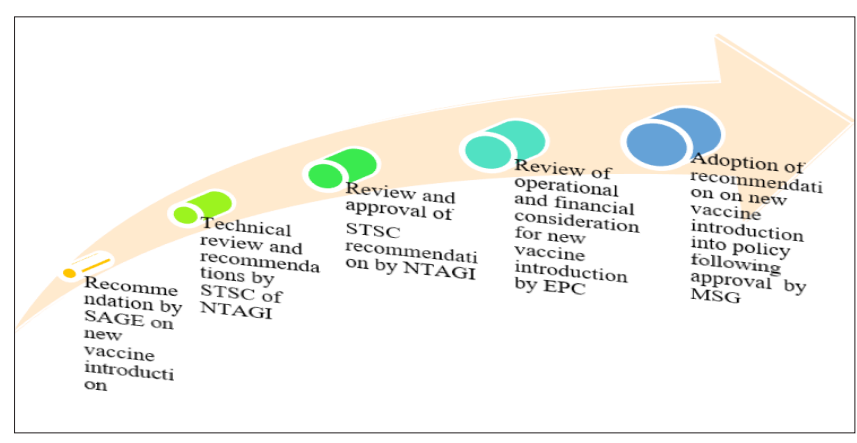

New Vaccine Introduction: A Regulatory Approval Process in India

Vaccine Expert Committee for the New Vaccine: Vaccine Expert Committee provides advice and develops plans \& strategies to introduce a new vaccine. This committee is a mix of domain experts which includes members from MoHFW, Indian Council of Medical Research (ICMR), developmental 
partners, and other experts. The expert committee guides in the development of technical specifications for the new vaccine, development of operational guidelines, and development of a strategy to ensure vaccine and logistics availability.

Vaccine Introduction Group: The group is constituted to support the introduction of new vaccines at ground level and consists of partner agencies (WHO, UNICEF, UNDP) and government bodies (NCCVMRC, NIHFW, Adverse Events Following Immunization (AEFI) Secretariat, etc). However, other partners support the activities as and when needed. Development and printing of guidelines \& training materials, coordination with the states, conducting training, vaccination-session monitoring, and reporting \& recording are primarily the responsibility of the lead partner agency such as WHO.
Pre-Introduction Phase/Preparatory Phase (i.e. after MSG Approval but before New Vaccine Introduction/ launch)

After the approval of the MSG, the preparation phase starts at least 3-6 months before the vaccine introduction or even before. Fund mobilization, development of a technical specification for vaccine, tender process \& procurement, cold chain assessment, vaccine \& logistics management, preparation of operational guidelines, IEC materials \& FAQ, human resource deployment, training need assessment, development of training package, capacity building exercise are carried out. Field level staffs are responsible for conducting headcount surveys, preparing duelist, micro-plan, communization plan, contingency plan, vaccine distribution plan, AVD plan, etc. and, ensure implementation of the plans as per Gol guidelines. ${ }^{9}$

\section{Major Activities Carried-out in the Preparatory Phase}

Funding support for new vaccine introduction: Funding may be made available either through Government budget or through donor support.

- Funding through the Government's health budget: For the vaccination program, the budgetary provision is made both at the center and state level. The Vaccine \& logistics procurement is centralized and being done by the national government. Under the State Program Implementation Plans (PIPs), the States propose operational cost and other funding support needed for IEC, communication, monitoring, and mobility support. The discussion of the state's PIPs is carried out during the National Program Coordination Committee (NPCC) meeting in MoHFW and based on the discussion with state officials; Ministry issues the "Record of Proceeding" (ROP) to the states highlighting NPCC decision and recommendation for funding support. Accordingly, financial allocations are made to States. ${ }^{10}$

- International Funding Support: BMGF is an international foundation supporting immunization services by providing funding support as well as technical insight. Similarly, Gavi - A global Vaccine Alliance is, an international organization, providing financial support for the introduction of a new vaccine. In 2018, Gavi had considered support to countries having average GNI per capita over the past three years equal to or below the threshold amount of US\$1,580. The eligibility threshold takes inflation into account and is updated on a yearly basis. As of now, Countries with a three-year average annual GNI per capita of \$1,630 or less are eligible to apply for Gavi assistance. Gavi provides support to eligible countries for Health System Strengthening (HSS), New Vaccine Support (NVS), Cold Chain Equipment Optimization Platform (CCEOP) and, Targeted Country Assistance (TCA) through its core partners (WHO, UNICEF, CDC, and the World Bank) and expanded partners (including local institutions) via Targeted Country Assistance (TCA). ${ }^{11}$

Development of Technical Specifications: The technical specifications contain item-wise details to ensure that vaccine meets the safety standards of India Pharmacopeia and India's Drugs and Cosmetics Act and Rules. As per the standard procedure vaccine, if manufactured in India, must meet the legal \& technical requirements provisioned under Drugs and Cosmetics Act 1940 and Rules 1945 amended from time to time. However, in the case of international vaccine manufacturers, the vaccine must meet the legal requirements of the respective country of origin but must be registered with the Drugs Controller General of India (DCGI). The technical specifications include information on dosage schedules, the route \& site of administration, dose packaging details (single dose or multi-dose vial), the temperature sensitivity of vaccines, shelf life, labeling for primary and secondary packaging, VVM monitor specifications, quality components and, regulatory compliance that is to be ensured by the supplier. Besides, it also includes pre-advice, airway bill, supplier's invoice, dispatch, packing list, lot release certificate etc. To ensure the quality of the vaccine, test protocol along with vaccine samples of all batches are sent to the head of the vaccine testing laboratory i.e. CDL, Himachal Pradesh as well. ${ }^{12,13}$ 
Vaccine Procurement and Cold Chain Management: Once the technical specifications are developed, the Empowered Procurement Wing of the MoHFW issues tender for centralized procurement of the vaccine and logistics as per GFR 2017. A reliable cold chain network is essential to ensure that vaccines are stored within the recommended temperature ranges $\left(2^{\circ} \mathrm{C}-8^{\circ} \mathrm{C}\right)$ to maintain vaccine quality, potency, and efficacy. Therefore, assessment of cold chain space, availability of stem cell thermometer and/or eVIN temperature logger, power-backup, and availability of contingency plan are other essential components.

Vaccine Stock, Transport and Logistics: Vaccine stock-out and wastage must not occur. Therefore, it is needed to have an estimation of the minimum stock and maximum stock of the vaccine \& logistics. Considering the lead time $\&$ to avoid stock out situations, the demand for the vaccine are raised by cold chain handlers ( $\mathrm{CCH}$ ) before vaccine reaches the minimum stock level.

Other Activities: Waste management, communication \& IEC, capacity building, training, supportive supervision, VPD surveillance and AEFI are essential activities that need to be assessed.

Preparedness Assessment Checklist for New Vaccine Introduction: Preparedness assessment checklists help in assessing and identifying strengths and weaknesses and to take corrective actions for the effective and successful introduction of any new vaccine. For example, the following 14 components (essential \& additional) were assessed before RVV introduction ${ }^{14}$ in India:

- Essential Components: Human resources vitals, background information, micro-planning status, training status, recording \& reporting practices, vaccine coverage and wastage, vaccine management, transport and logistics, waste management and injection safety, monitoring and evaluation, Adverse Events Following Immunization, mobilization, advocacy, and communication, surveillance, and cold chain maintenance.

- Additional Components: General impressions and additional remarks/ comments.

\section{Vaccine Introduction/ Vaccine Launch Phase}

After the preparation phase and preparedness assessment, the official launch of the vaccine takes place using a platform of national Training of Trainers (ToT), state-level orientation workshops, and media briefing.

Further, State level workshop is conducted to orient districtlevel officials and partners who in-turn provides training to block-level staff and Field Level Workers (FLWs). State Task Force on Immunization (STFI) and District Task Force on Immunization (DTFI) is responsible for monitoring new vaccine introduction, providing suitable recommendations, and preparing an action plan to address gaps/issues. Delivery of vaccine and logistics from higher vaccine stores to Cold Chain Points (CCP) are carried out well in advance. Besides, printing (in local language) and dissemination of new vaccine guidelines, reporting formats, and IEC materials are mostly carried-out by States before the new vaccine introduction. An effective communication plan supports FLWs in the mobilization of targeted beneficiaries and helps in overcoming vaccine hesitancy issues. Data collection, data analysis, recording and reporting are also carried out on daily basis to assess the coverage. One of the major activities during the introduction is to monitor and respond to any adverse event reported after vaccination. AEFI can either be a true adverse event i.e. a result of the vaccine process or a coincidental event which is not due to the vaccine or the vaccination process. Therefore, before bringing any new vaccine in the system, strengthening of existing vaccine-preventable disease and $A E F I$ surveillance system and capacity building of staff must be carried out. .,14,15 $^{2}$

\section{Post-Introduction and Expansion Phase}

The Gol uses the Health Management Information System (HMIS) and other reported data to review the service delivery component of new vaccination. States upload monthly data of proceeding month on the HMIS portal as per the ongoing schedule i.e. either on or before the 20th of every month. For instance, under UIP, the immunization data from HMIS provides FIC, coverage data for each antigen, status of AEFI cases (abscess, death, others), number of immunization sessions (held vs. planned) and number of VPDs reported, etc. The Mother and Child Tracking System (MCTS) portal also provides antigen wise coverage against registered children. At initial phases, the data for new vaccine coverage may be collected through manual reporting form from states. Concurrent monitoring is another source of immunization data.

Expansion of new vaccine also needs due consideration because the new vaccine introduction is mostly being done on a pilot basis or phased manner depending on various factors such as vaccine availability, disease burden, state government's priority, as well as, financial implications. Further, Post Introduction Evaluation (PIE) helps in evaluating the impact of the new vaccine in reducing disease burden and improving vaccination coverage. PIE has three components: desk review \& adaption of the PIE tool; fieldwork, and compilation of data reporting 
and; formulation of recommendations. PIE is generally conducted using a questionnaire, checklists, record reviews, and observation of practices at all health facilities and for all thematic areas of vaccination. The findings of the post-introduction evaluation, field visits, and research studies are taken, as and when required, to arrive at the final decision to expand the geographical coverage of the new vaccine..$^{5,14,15}$

\section{Compassionate Use/ Expanded Access}

US FDA defines the term 'compassionate use' also called 'expanded access' as a "potential pathway for a patient with an immediately life-threatening condition or serious disease or condition to gain access to an investigational medical product (drug, biologic, or medical device) for treatment outside of clinical trials when no comparable or satisfactory alternative therapy options are available".

An investigational product that is in development and testing but has not been approved by the FDA. These products must be primarily used as part of a clinical trial. However, if it is not possible for a patient to be a part of clinical trials due to reasons like no ongoing trials, no eligibility or limited access to participate in clinical trials, etc. expanded access/ compassionate use may be considered for a patient to get an investigational product. Compassionate use has similarities with clinical trials, but the data collection process is different. The government also approves Compassionate use of drugs, biologicals \& devices during the critical situation to reduce the time-lag between the end of clinical trial studies and potential regulatory approvals required before ensuring investigational product's market availability and use in the community. However, informed consent are required. It is also pertinent to consider potential risks and the potential benefits of investigational drugs, biologics, devices, and be aware that limited information available about the safety or efficacy of the investigational product may lead to adverse effects, hospitalization, and reduced life expectancy. ${ }^{16}$

\section{Emergency Use Authorization}

Emergency use authorization allows the use of an investigational drug or biological product on a human subject in a life-threatening situation in which no standard acceptable treatment is available, and in which there is not enough time to obtain regulatory approval. However, the investigator is required to obtain the informed consent of the subject or the subject's legally authorized representative. FDA defines a life-threatening situation that includes the scope of both life-threatening i.e. diseases or conditions where the likelihood of death is high and severely debilitating i.e. diseases or conditions that cause major irreversible morbidity. The ethical consideration and criteria like life-threatening or severely debilitating situation, lack of standard treatment method, unproven efficacy, insufficient time to obtain regulatory review and approval, informed consent associated with the emergency use of investigational products must be taken into consideration before approving emergent use of an experimental treatment. Consent waiver may be granted if the patient has life-threatening status, communication with the patient cannot be done and there is no time to wait for consent from the legal representative of the patient and there are no approved alternative drugs, biologics and devices are available which can enhance chances of saving the participant's life. ${ }^{17}$

\section{Discussion}

The above-explained SOPs for new vaccine introduction in India indicates that even after vaccine development after successful clinical trials (having evidence of safety \& efficacy of the investigational product and approval by regulatory agencies), there are extensive reviews, discussions, and studies by technical bodies like STSC \& NTAGI before submitting the new vaccine recommendations to MSG for final approval. The process is certainly time-consuming and may not be feasible in the current scenario of COVID-19 pandemic because i. No vaccines with proven efficacy are developed \& available now, ii. Vaccine introduction and approval process takes 2-3 years after satisfactory vaccine development.

For example, NTAGI has discussed the agenda item on 'Potential introduction of RVV in UIP' in September $2013^{18}$ and referred the matter to STSC to review the evidence, but the Rota Virus Vaccine had been introduced in a phased manner in March 2016, leading to time gap in decision making \& vaccine use of about 2.5 years. During PCV introduction, NTAGI recommended a phased introduction of PCV under the agenda 'Potential introduction of Pneumococcal Conjugate Vaccines (PCVs) in India's Universal Immunization Programme' in August 2015. ${ }^{19}$ But MSG approved the NTAGI \& EPC recommendations for PCV introduction in identified five states (Bihar, Madhya Pradesh, Himachal Pradesh, and Parts of Uttar Pradesh and Rajasthan) in December 2016 after considering all aspects and ensuring the safety of PCV use in the population. Similarly, the NTAGI reviewed agenda item 'diphtheria disease burden in India' and endorsed replacement of TT vaccine with Td vaccine in India's immunization program for all age groups, including pregnant women in Dec $2016^{20}$ but actual replacement of TT with Td vaccine could be taken place in 2019, primarily due to extensive preparatory activities and after consuming existing TT stocks.

These examples reflect the Indian government's commitment for providing safe and quality vaccines to all without compromising product efficacy, safety \& quality, therefore, time-consuming studies to gather safety \& efficacy evidence are carried out. But these decisions were taken 
when there was enough time/time-flexibility, availability of approved vaccine, presence of alternative vaccine and many vaccine suppliers in markets for comparative studies, and most importantly there was no pandemic \& life-threatening situation as we are facing during the COVID-19 pandemic.

To understand the COVID-19 vaccine introduction in the pandemic situation, we need to understand some vital criteria (similar to EUA/compassionate use) that may have a direct bearing on the decision about the way COVID-19 vaccine can be used. These are:

- COVID-19 is a life-threatening disease, causing high mortality \& morbidity globally

- Expected high time-lag between the end of clinical trial studies and standard process of regulatory approvals for vaccine use

- There is no comparable or satisfactory treatment

- Unproven safety and efficacy data of COVID-19 drugs/ vaccines, which are still under various phases of a clinical trial

- Frequent changes in COVID-19 disease characteristics/ symptoms

- Developing Herd Immunity against COVID-19 is challenging

- Only social distancing measures, maintaining hygiene (hand wash/sanitization), and the use of personal protective equipment (PPEs) are possible ways available to get protection (not cure) against the disease

These characteristics are mostly fulfilling the compassionate use \& EUA criteria. Herd Immunity i.e. indirect protection from SARS-COV-2 coronavirus is another agenda being discussed globally. We may get protected from the disease by developing herd immunity and becoming immune to the disease only if we had any vaccination or previous infection. Now, in the COVID-19 situation, infecting large populations \& putting them at high risk is not an easy decision for the government and not advisable also, and we do not have any vaccine as well so achieving protection through herd immunity is very challenging.

We also need to understand that even-though many vaccine candidates are under development and are at various phases of clinical trials, however, the scientific data collection may require a greater number of participants because of the long incubation period, differed COVID-19 symptoms (loss of appetite, muscle pain, fatigue, headache, diarrhea, shortness of breath, pneumonia, loss of taste $\&$ smell), varied infected age group, distinct types of SARSCoV-2 coronavirus causing disease and other associated complications (Liver Injury, Cytokine syndrome, Respiratory Failure, Acute Respiratory Distress Syndrome, Mental Health issues, Non Communicable diseases) and many more.

Now, the question comes - Is it possible to get participants with such varied symptoms under clinical trial studies? Is clinical research of new vaccine could be done with limited participants? How the safety \& efficacy parameters could be fulfilled for patients reporting different symptoms of the same disease? Thus, the COVID-19 vaccine study may be challenging \& time-consuming especially when there is no proven treatment and, the pandemic is taking lives at a very fast pace.

In India, convalescent plasma therapy has been approved for compassionate use and therefore, plasma banks are being set up to ensure round the clock availability. Compassionate use of drugs may help us in the development of a proven treatment or vaccine. ${ }^{21}$ Similarly, Ebola virus disease outbreak had occurred in West Africa in August 2014. Like COVID-19, Ebola can also be transmitted if people come in direct contact with an infected animal (bat or nonhuman primate) or a sick or dead Ebola-infected person. U.S. FDA authorized emergency use of the first Ebola fingerstick test with a portable reader. ${ }^{22}$ Also, there was no available treatment or vaccines so after considering the access and ethical implications, WHO decided that "it is ethical to offer unproven interventions with as yet unknown efficacy and adverse effects, as potential treatment or prevention". Thus, the rVSV-ZEBOV vaccine that was not licensed, had been used under "expanded access" or "compassionate use" research studies. Volunteers took part in the study with consent, and they were followed up after vaccination to monitor safety. Later the vaccine is proved effective in treating Ebola in study population and subsequently, saved many lives. ${ }^{23}$

Therefore, compassionate use and emergency use authorization may be allowed for drug/vaccine efficacy studies and possible COVID-19 treatment, only if the country's government, manufacturers, physicians, and research agencies meet safety standards, take mandatory ethical approvals/ethical clearance as well as, participant's consent into account. Further, in addition to emergency use approval, all phases of clinical trials of vaccine on human may be carried out as per the standard protocol, rules \& regulations, without compromising on vaccine efficacy \& other standards and by devoting the time needed to get a safe vaccine in future. For the time-being, until we have any proven vaccine, we all need to strictly follow social distancing measures, use of personal protective equipment, hand hygiene etc. to avoid further transmission of coronavirus and prevent community spread.

\section{Conclusion}

COVID-19 disease burden, mortality \& morbidity rates, high rate of global transmission of the disease and economic slowdown are some important factor that has brought Countries on a single platform to introduce new COVID-19 vaccine as soon as possible. Further, continued discussion 
on compassionate use \& emergency use authorization by the Government for administering unproven investigational treatment has left everyone bewildered. The incomplete information and knowledge of the technical concepts may hamper understanding of the common public about the Government's decisions and may lead to a rumor if not addressed well in time. We need to understand that, worldwide, no government or scientific fraternity want to put the lives of their citizen/global community at risk by compromising safety, efficacy, and ethical parameters. Therefore, the article is an attempt to provide information, to all non-technical person, other stakeholders, students and individuals living in the global family, about various clinical terms and regulatory processes related to COVID-19 vaccine introduction and how they play a key role in decision making so that everyone can see the real picture with clear lenses and make the right decision at right time.

\section{Acknowledgement}

I would like to acknowledge the efforts being made by the government officers, policymakers, vaccine manufacturers, scientists, and most importantly our field level Corona Warriors - District Administrators, Doctors, Nurses, Police officials and Sanitation Workers.

\section{Conflict of Interest: None \\ References}

1. Worldometer. COVID-19 Coronavirus Pandemic. 2 Aug 2020. Available from: https://www.worldometers.info/ coronavirus/.

2. Centers for Disease Prevention and Control. Vaccine Testing and the Approval Process. Available from: https://www.cdc.gov/vaccines/basics/test-approve. html.

3. US Food and Drug Administration. Clinical Research. Available from: https://www.fda.gov/ patients/drug-development-process/step-3-clinicalresearch.

4. Centers for Disease Prevention and Control. Vaccine Testing and the Approval Process. Available from: https://www.cdc.gov/vaccines/parents/infographics/ journey-of-child-vaccine-h.pdf.

5. World Health Organization. Vaccine Introduction Guidelines. Geneva: Department of Vaccines and Biologicals; 2005. Available from: https://www.who. int/immunization/hpv/plan/vaccine_introduction_ guidelines_who_2005.pdf.

6. Ministry of Health and Family Welfare, Government of India. Code of Practice; July 2015. Available from: https://main.mohfw.gov.in/sites/default/files/CODE_ OF_PRACTICE.pdf.

7. National health mission, Ministry of health and family welfare, Government of India. Institutional
Mechanism. Delhi: 2020. Available from:https://nhm.gov. in/index1. php?lang=1\&level=0\&linkid=456\&lid=685.

8. Ministry of health and family welfare, Government of India. National operational guidelines for introduction of Pneumococcal Conjugate Vaccine. Delhi: 2017. Available from: https://nhm.gov.in/New_Updates_2018/ NHM_Components/Immunization/Guildelines_for_ immunization/Operational_Guidelines_for_PCV_ introduction.pdf.

9. World Health Organization. Vaccine Introduction Guidelines. Geneva: Department of Vaccines and Biologicals; 2005. Available from: https://www.who. int/immunization/hpv/plan/vaccine_introduction_ guidelines_who_2005.pdf.

10. National health mission, Ministry of health and family welfare, Government of India. NHM - STATE ANNUAL PLAN. Available from: https://nhm.gov.in/index4. php?lang=1\&level=0\&linkid=449\&lid=53.

11. Gavi - the vaccine alliance. How to request new GAVI support. Oct, 2019. Available from: https://www.gavi. org/sites/default/files/document/support/How-torequest-new-Gavi-support-for-2020.pdf.

12. Ministry of health and family welfare, Government of India. National operational guidelines for introduction of measles-rubella vaccine. Delhi: 2017. Available from: http://origin.searo.who.int/india/topics/measles/ measles_rubella_vaccine_guidelines.pdf.

13. Ministry of health and family welfare, Government of India. Guidelines on Universal Immunization Program. [Online] Delhi: Available from: https://main. mohfw.gov.in/sites/default/files/5628564789562315. pdf.

14. Ministry of health and family welfare, Government of India. National operational guidelines for introduction of rotavirus vaccine in UIP. Delhi: March 2019. Available from: https://nhm.gov.in/New_Updates_2018/ NHM_Components/Immunization/Guildelines_ for_immunization/Operational_Guidelines_for_ Introduction_of_Rotasiil_in_UIP.pdf.

15. Ministry of health and family welfare, Government of India. National operational guidelines for introduction of measles-rubella vaccine. Delhi: 2017. Available from: http://origin.searo.who.int/india/topics/measles/ measles_rubella_vaccine_guidelines.pdf.

16. U.S. FDA, Expanded Access: Information for Physicians. April 2020. Available from: https://www.fda.gov/newsevents/public-health-focus/expanded-access.

17. Balamuralidhara V, Vaishnav AM, Bachu V, Pramod TM. Emergency Use Authorization: At Zero Hour. International Journal of Drug Regulatory Affairs 2018; 3: 18-23. 10.22270/ijdra.v3i4.173. Available from: https:// www.researchgate.net/publication/326597334_ EMERGENCY_USE_AUTHORIZATION_AT_ZERO_HOUR . 
18. Ministry of health and family welfare, Government of India. Minutes of the Meeting of the National Technical Advisory Group on Immunization (NTAGI). Sep. 2013. Available from: https://www.nitag-resource. org/sites/default/files/ab996b76c18bf71851b7ebfae9 bb493055cf00e0_1.pdf.

19. Ministry of health and family welfare, Government of India. Minutes of the Meeting of the National Technical Advisory Group on Immunization (NTAGI). Aug 2015. Available from: https://main.mohfw.gov. in/sites/default/files/29914318411467722384.pdf.

20. Ministry of health and family welfare, Government of India. Minutes of the Meeting of the National Technical Advisory Group on Immunization (NTAGI). Dec 2016. Available from: https://main.mohfw.gov. in/sites/default/files/48798238911486636162.pdf.

21. Press information bereau, Ministry of health and family welfare. Every single donor counts in our journey towards victory over COVID-19 and we need more and more of these CORONA WARRIORS: Dr Harsh Vardhan Posted On: 19 JUL 2020 7:07PM by PIB Delhi. Available from: https://pib.gov.in/ PressReleasePage.aspx?PRID=1639826.

22. U.S. food and drug administration. FDA authorizes emergency use of first Ebola fingerstick test with portable reader. Available from: https://www.fda.gov/ news-events/press-announcements/fda-authorizesemergency-use-first-ebola-fingerstick-test-portablereader.

23. World health organization. Ebola then and now. Eight lessons from West Africa that were applied in the Democratic Republic of the Congo. 2019 Weblog. Available from: https://www.who.int/news-room/ feature-stories/detail/ebola-then-and-now. 